\title{
Über das Nadelwadhstum bei der Oxydation von Kupfer. II
}

\author{
Von Ludwig Albert und Walther Jaenicke
}

\author{
Aus dem Laboratorium für Elektronenmikroskopie und dem Institut für Physikalische Chemie \\ und Elektrochemie der Technischen Hochschule Karlsruhe \\ (Z. Naturforschg. 15 a, 59-65 [1960]; eingegangen am 10. August 1959)
}

\begin{abstract}
Es wird gezeigt, daß etwa $75 \%$ der während der Oxydation von Kupfer bei $500{ }^{\circ} \mathrm{C}$ insgesamt entstehenden Nadeln von der gleichzeitig wachsenden Oxydschicht zugedeckt werden, da die meisten infolge von Inhibition nur geringe Längen erreichen. Die Längenverteilung der Nadeln wird dagegen durch das Schichtwachstum nur unwesentlich beeinflußt.

Der mit zunehmender Oxydationszeit schwächere Abfall der Längenverteilungskurven wird unter der Annahme gedeutet, daß inhibierende Fremdstoffe aus dem Kupfer in der wachsenden Deckschicht absorbiert werden.

Dickenverteilung und Dickenwachstum werden diskutiert. Beides beeinflußt die Längenverteilung nicht, wenn die Inhibitionswahrscheinlichkeit unabhängig vom Nadelquerschnitt ist.
\end{abstract}

Im ersten Teil der vorliegenden Arbeit ${ }^{1}$ (im folgenden mit I bezeichnet) wurde die Längenverteilung von Oxydnadeln, die bei der Oxydation von Kupfer auftreten, bestimmt und unter vereinfachenden Voraussetzungen gedeutet. Dabei wurde das gleichzeitig mit dem Nadelwachstum einhergehende Schichtwachstum unberücksichtigt gelassen und alle Nadeln als gleich dick angenommen. Von diesen Vernachlässigungen wird im folgenden abgesehen und ferner der verschieden starke Abfall der Längenverteilungskurven bei verschiedenen Oxydationszeiten begründet.

In Tab. 1 sind die in Teil I und II der Arbeit benutzten Symbole zusammengestellt und erläutert.

\footnotetext{
$T$ = Oxydationsdauer der Probe (Versuchszeit)

$t=$ laufende Zeit, vom Versuchsbeginn an gerechnet

$\tau=$ laufende Zeit, vom Entstehungszeitpunkt der Nadeln

an bis zum Ende des Längenwachstums gerechnet

$L=$ beobachtete Nadellänge nach Abschluß des Versuchs

$l=$ während des Nadelwachstums über die gleichzeitig wachsende Oxydschicht hinausragende Nadellänge

$\lambda$ = Nadellänge, vom Entstehungsort aus gerechnet, welcher durch Schichtwachstum zugedeckt wird

$\delta=$ Oxydschichtdicke zur Zeit $t$ bzw. $t+\tau$

$N=$ Gesamtzahl der nach Abschluß des Versuchs beobachteten Nadeln auf einer Fläche $F$ der Oxydschicht

$\mathrm{d} n_{0}=$ Zahl der zur Zeit $t$ im Zeitelement $\mathrm{d} t$ entstehenden Nadeln

$\mathrm{d} n=$ Zahl der Nadeln, die zur Zeit $t$ im Zeitelement $\mathrm{d} t$ entstanden sind und zur Zeit $t+\tau$ noch weiterwachsen

$q=$ Nadelquerschnitt

$s \quad=$ Nadelumfang.
}

Tab. 1. Benutzte Symbole.

\section{A. Einfluß des Schichtwachstums auf die Längenverteilung der Nadeln}

Der Zusammenhang zwischen der Länge $\lambda$ und der Wachstumszeit $\tau$ der Nadeln für geschwindig- keitsbestimmende Volumen- oder Oberflächendiffusion $\lambda=a \sqrt{\tau}$ gilt in dieser Form nur dann, wenn das Wachstum der Oxydschicht vernachlässigt werden kann, d. h. wenn die Oxydschicht wesentlich langsamer wächst als die Nadeln. Auch für das Schichtwachstum gilt - von den kürzesten Zeiten abgesehen - mit guter Annäherung ein parabolischer Zusammenhang ${ }^{2}$ zwischen der Oxydationszeit $t$ und der Schichtdicke $\delta$. Unter etwa $2 \mathrm{~min}$ wächst $\delta$ etwas weniger als proportional mit $t$. Trotzdem soll von den kürzesten Zeiten an $\delta=b \sqrt{t}$ gesetzt werden, um den Einfluß des Schichtwachstums auf das Wachstumsgesetz der Nadeln und deren Längenverteilung abzuschätzen.

Das Schichtwachstum verkürzt einerseits die $\mathrm{Na}$ dellänge, andererseits werden die Diffusionswege $l$ zur Nadelspitze kleiner, so daß die Nadeln schneller wachsen. In Abb. 1 a sind die Verhältnisse für eine noch wachsende Nadel dargestellt. Abb. 1 b zeigt die Zusammenhänge nach Beendigung der Oxydation zur Zeit $T$. Aus Abb. 1 a ergibt sich für die differentielle Längenzunahme einer Nadel

$\mathrm{d} l=\mathrm{d} \lambda-\mathrm{d} \delta$ oder $\quad \mathrm{d} l=\frac{a^{2} \mathrm{~d} \tau}{2 l}-\frac{b \mathrm{~d} \tau}{2 \sqrt{t+\tau}}$.

Ohne Berücksichtigung des Schichtwachstums ist $b=0$ und $l=\lambda$. Die Nadellänge hängt also in diesem Fall nur von der Wachstumszeit $\tau$ ab. Die Differentialgleichung (1) zeigt aber, daß wegen des Schichtwachstums die Nadellänge auch von ihrer Entstehungszeit $t$ abhängt. Die Lösung dieser Differentialgleichung führt auf eine sehr verwickelte

1 L. Albert u. W. Jaenicke, Z. Naturforschg. 14 a, 1040 [1959]

- W. Jaenicke u. S. Leistikow, Z. Phys. Chem., N.F. 15, 175 [1958]. 

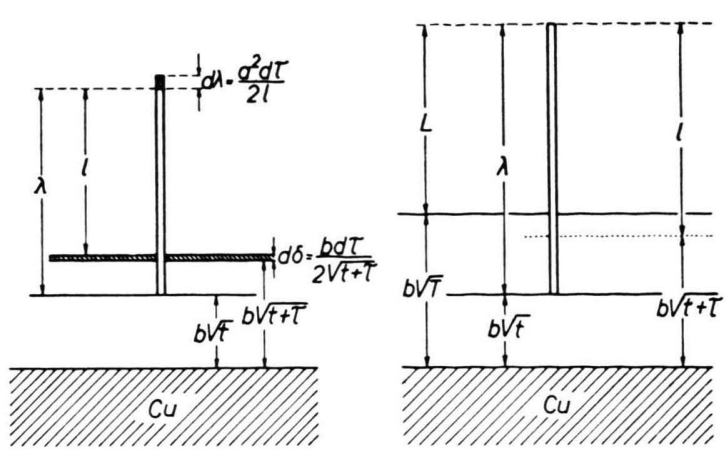

Abb. 1. Zusammenhang zwischen Nadellänge und Schichtwachstum, a) für eine noch wachsende Nadel (links), b) nach Beendigung der Oxydation (rechts).

Formel, aus der sich weder $l$ noch $\tau$ explizit darstellen lassen.

In die in I berechnete Längenverteilung, deren Form im wesentlichen durch die Inhibitionsvorgänge bestimmt wird, geht das Wachstumsgesetz nur wegen der Nadeln in die Rechnung ein, die bei Beendigung der Oxydation noch wachstumsfähig waren [vgl. Gl. $(\mathrm{I}, 6)]$. Die Entstehungszeiten dieser Nadeln sind $t=T-\tau$. Um die Längenverteilung der Nadeln zu gewinnen, genügt es daher, die Differentialgleichung (1) für $t=T-\tau$ zu lösen.

Den Zusammenhang zwischen der beobachteten Länge $L$, der ,wirklichen“ Nadellänge $\lambda$ und der über die Schichtoberfläche hinausragenden Länge $l$, welche die Nadel in dem Augenblick hat, in dem sie aufhört zu wachsen, liest man aus Abb. 1 b ab:

$$
L+b \sqrt{T}=\lambda+b \sqrt{t}=l+b \sqrt{t+\tau} ;
$$

$t$ ist der Entstehungszeitpunkt der Nadel und $\tau$ die Zeit, während welcher die Nadel wächst.

Es ist $\mathrm{d} \lambda=a^{2} \mathrm{~d} \tau / 2 l(\tau)$. Für alle Nadeln, die ihr Wachstum zur Zeit $t=T-\tau$ beginnen und zur Zeit $T$ infolge Abbruchs des Versuchs beenden, gilt mit Gl. (2)

$$
\lambda=\int_{0}^{\tau=T-t} \frac{a^{2}}{2 l(\tau)} \mathrm{d} \tau=L+b \sqrt{ } T-b V t
$$

oder

$$
\lambda=I(T-t)-I(0)=L+b \sqrt{T}-b \sqrt{ } T-(T-t) .
$$

Durch Differentiation folgt

$$
\frac{\mathrm{d} \lambda}{\mathrm{d}(T-t)}=\frac{\mathrm{d} I(T-t)}{\mathrm{d}(T-t)}=\frac{\mathrm{d} L}{\mathrm{~d}(T-t)}+\frac{b}{2 \sqrt{ }(T-(T-t)}
$$

oder $\quad-\frac{\mathrm{d} \lambda}{\mathrm{d} t}=\frac{a^{2}}{2 l(T-t)}=-\frac{\mathrm{d} L}{\mathrm{~d} t}+\frac{b}{2 \sqrt{ } t}$.

$l_{(T-t)}$ ist aber gleich $L$, so daß man für die zu den
Zeiten $t=T-\tau$ entstehenden Nadeln

$$
-2 \frac{\mathrm{d} L}{\mathrm{~d} t}=\frac{a^{2}}{L}-\frac{b}{V t}
$$

oder mit $L$ aus Gl. (2)

$$
-\frac{a^{2}}{2} \frac{\mathrm{d} t}{\mathrm{~d} \lambda}=\lambda-b V T+b V t
$$

erhält.

Substitution von $x=\lambda-b \sqrt{T}$ und $y=V t$ in Gl. (4) ergibt mit $\mathrm{d} \lambda=\mathrm{d} x$ und $\mathrm{d} t=2 y \mathrm{~d} y$ :

$$
-a^{2} \frac{\mathrm{d} y}{\mathrm{~d} x}=\frac{x}{y}+b .
$$

eine homogene Differentialgleichung 1. Ordnung, die sich durch die weitere Substitution $x / y=u$ oder $x=u y$ lösen läßt. Man erhält mit $\mathrm{d} x=u \mathrm{~d} y+y \mathrm{~d} u$

$$
-\frac{\mathrm{d} y}{y}=\frac{u+b}{u^{2}+b u+a^{2}} \mathrm{~d} u
$$

und, da $\frac{1}{4} b^{2}-a^{2}<0$ ist (die Nadeln wachsen schneller als die Schicht), nach Integration

$$
\begin{aligned}
-\ln y=\frac{1}{2} \ln \left(u^{2}+\right. & \left.b u+a^{2}\right) \\
& +\frac{\frac{1}{2} b}{\sqrt{a^{2}-\frac{1}{4}} b^{2}} \text { arctg } \frac{u+\frac{1}{2} b}{\sqrt{a^{2}-\frac{1}{4} b^{2}}}+C
\end{aligned}
$$

oder

$$
\begin{array}{r}
-C=\ln \sqrt{(\lambda-b V T)^{2}+b \sqrt{ } t(\lambda-b \sqrt{ } T)+a^{2} t} \\
+\frac{b}{\sqrt{4 a^{2}-b^{2}}} \operatorname{arctg} \frac{2 \frac{\lambda-b \sqrt{ } T}{\sqrt{ } t}+b}{\sqrt{ } 4 a^{2}-b^{2}} .
\end{array}
$$

Die Integrationskonstante $C$ ergibt sich aus der Bedingung, daß für $\tau=0$ (oder $t=T-\tau=T$ ) $\lambda=0$ ist:

$$
-C=\ln a \sqrt{ } T+\frac{b}{\sqrt{4 a^{2}-b^{2}}} \operatorname{arctg} \frac{-b}{\sqrt{4} a^{2}-b^{2}} .
$$

Da $\quad \operatorname{arctg}(-A)=-\operatorname{arctg} A$

und $\quad \operatorname{arctg} A+\operatorname{arctg} B=\operatorname{arctg} \frac{A+B}{1-A B}$

ist, erhalten wir als Lösung der Diffgl. (4)

$$
\begin{aligned}
& \ln \sqrt{\frac{(\lambda-b \sqrt{ } T)^{2}+b \sqrt{t}(\lambda-b \sqrt{T})+a^{2} t}{a^{2} T}} \\
& +\frac{b}{\sqrt{ } 4 a^{2}-b^{2}} \operatorname{arctg} \frac{\sqrt{4 a^{2}-b^{2}(\lambda-b V T+b \sqrt{ } t)}}{2 a^{2} \sqrt{ } t-b(\lambda-b \sqrt{ } T+b \sqrt{t})}=0 .
\end{aligned}
$$

Die beobachtbare Nadellänge ist aber nicht $\lambda$, sondern $L$. Substitution von $L=\lambda-b \sqrt{T}+b \sqrt{ } t$ aus Gl. (2) in Gl. (5) ergibt

$$
\begin{aligned}
& \ln \sqrt{\frac{L(L-b \sqrt{t})+a^{2} t}{a^{2} T}} \\
& +\frac{b}{\sqrt{ } 4 a^{2}-b^{2}} \operatorname{arctg} \frac{L \sqrt{4 a^{2}-b^{2}}}{2 a^{2} \sqrt{ } t-b L}=0
\end{aligned}
$$


Gl. (6) ist zugleich die Lösung der Diffgl. (3).

Bei Vernachlässigung des Schichtwachstums $(b=0$, $L=\lambda$ ) ergibt sich aus Gl. (5) oder (6) als Spezialfall die einfache Gleichung $\lambda=a \sqrt{T-t}$, also der in I benutzte parabolische Zusammenhang zwischen Länge $\lambda$ und Wachstumszeit $\tau=T-t$ der bei $\mathrm{Be}$ endigung der Oxydation noch wachstumsfähigen $\mathrm{Na}$ deln. Mit Berücksichtigung des Schichtwachstums tritt Gl. (5) bzw. (6) an die Stelle von $\lambda=a \sqrt{T-t}$.

Die arc tg in Gl. (5) und (6) durchlaufen von $t=T, \lambda=0$ bzw. $L=0$ beginnend bis $t=0$ den Bogen des Einheitskreises von Null aus in positiver Richtung.

Durch Gl.(5) ist die Entstehungszeit $t$ der Nadeln gegeben, die ohne Störung durch Inhibition bei Beendigung der Oxydation zur Zeit $T$ gerade die Länge $\lambda$ erreichen. Für diese Nadeln ist der Zusammenhang zwischen $t$ und $\lambda$ bei einer vorgegebenen Oxydationszeit $T$ durch die stark ausgezogene Grenzkurve in Abb. 2 a dargestellt. Alle anderen Nadeln, die die gleiche Länge $\lambda$ erreichen, sind zu einer früheren Zeit $t$ entstanden und durch Inhibitionsvorgänge am Weiterwachsen gehindert worden. Die Grenzkurve [Gl. (5)] schneidet die $\lambda$-Achse $(t=0)$ bei

$\lambda_{\max }=b \sqrt{T}+a \sqrt{T} \cdot \exp \left[\frac{-b}{\sqrt{4 a^{2}-b^{2}}} \operatorname{arctg} \frac{\sqrt{4 a^{2}-b^{2}}}{-b}\right]$.

Die Zahl $|\mathrm{d}[\mathrm{d} n]|$ der inhibierten Nadeln, welche zur Zeit $t$ im Zeitelement $\mathrm{d} t$ entstehen und Längen zwischen $\lambda$ und $\lambda+\mathrm{d} \lambda$ erreichen, soll in einem Flächenelement $\mathrm{d} t \mathrm{~d} \lambda$ der Abb. 2 a enthalten sein. Sie ist gegeben durch die in I aufgestellte Gl. (5) :

$$
|\mathrm{d}[\mathrm{d} n]|=k \frac{c}{\sqrt{t}} e^{-k \lambda} \mathrm{d} t \mathrm{~d} \lambda .
$$

$c / \bar{t}$ ist die Keimbildungsgeschwindigkeit und $k$ ein $\mathrm{Ma} ß$ für die Inhibition ( $k$ wurde in I Inhibitionsfaktor genannt).

Um alle Nadeln zu erfassen, die diese Länge $\lambda$ besitzen, muß Gl. (I, 5) längs des gestrichelt eingezeichneten Weges von $t=0$ bis zur Grenzkurve $t=t_{2}$ integriert und zu dem Integral noch die Anzahl der Nadeln hinzugefügt werden, die zu der durch Gl.(5) gegebenen Zeit $t=t_{2}$ entstanden sind:

$$
\mathrm{d} N=\int_{0}^{t_{2}} k e^{-k \lambda} \mathrm{d} \lambda \frac{c}{\sqrt{t}} \mathrm{~d} t+\frac{c}{\sqrt{t_{2}}} e^{-k \lambda} \mathrm{d} t_{2} .
$$

Das zweite Glied ist die Nadelzahl, mit welcher die Grenzkurve besetzt zu denken ist.
Nun interessiert aber nicht die $\lambda$-Verteilung, sondern die Verteilung der beobachteten Nadellängen $L$. $\mathrm{Zu}$ diesem Zweck muß aus Gl. (2) $\lambda=L+b \sqrt{T}-b \sqrt{t}$ in die Gl. (8) substituiert und diese so über $t$ integriert werden, daß dabei $L$ konstant bleibt: Der Integrationsweg ist die in Abb. 2 a strichpunktiert eingezeichnete Kurve

$$
\lambda=L+b \sqrt{T}-b \sqrt{t} .
$$

Für $L=0$ ist der Integrationsweg die dazu parallel verschobene und ausgezogen eingezeichnete Kurve $\lambda=b(V T-V t)$. Das links dieser Kurve gelegene
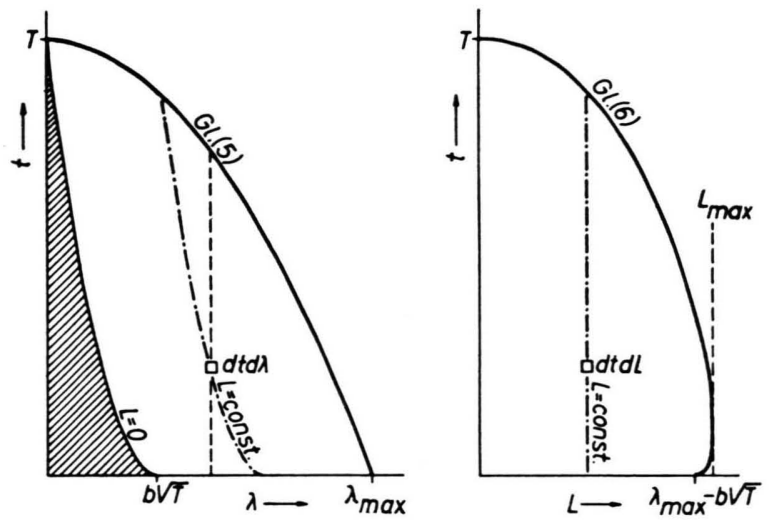

Abb. 2 a und 2 b. Zur Berechnung der Längenverteilung der Nadeln. Erklärung im Text.

schraffierte Gebiet beinhaltet alle die Nadeln, welche von der Oxydschicht zugedeckt werden und sich infolgedessen der Beobachtung entziehen.

Statt längs der strichpunktierten Kurve zu integrieren, kann man aber auch alle Punkte in Abb. 2 a um das Stück $b(\sqrt{T}-\overline{V t})$ nach links verschieben und erhält so die Abb. 2 b. Der Integrationsweg für gleiche Längen $L$ läuft jetzt parallel zur $t$-Achse und das Nadeln enthaltende Gebiet wird begrenzt durch die Funktion (6).

Man erkennt aus Abb. 2 b, daß die maximale Länge $L_{\max }$ infolge des Schichtwachstums nun von Nadeln erreicht wird, welche zu einer etwas späteren Zeit als $t=0$ entstehen und bis zum Ende der Oxydationszeit $T$ ununterbrochen wachsen. Diese maximal mögliche Länge $L_{\max }$ ergibt sich mit $\mathrm{d} L / \mathrm{d} t=0$ aus Gl. (3) und (6) zu

$$
L_{\max }=a \sqrt{T} \cdot \exp \left\{\frac{-b}{\sqrt{4 a^{2}-b^{2}}} \operatorname{arctg} \frac{\sqrt{4 a^{2}-b^{2}}}{b}\right\} .
$$

Sie ist also trotz des Schichtwachstums weiterhin proportional $\sqrt{T}$. Nach den Messungen von JAENICKE und 
Leistikow ${ }^{2}$ ist bei $500{ }^{\circ} \mathrm{C} b=(0,5 \pm 0,1) \mu / \mathrm{min}^{1 / 2}$. Setzt man diesen Wert in Gl. (9) ein, dann erhält man den durch die Beobachtung nahegelegten Wert $L_{\max } \approx 2 \sqrt{(T / \min )} \mu$, wenn $a=5 b \approx 2,5 \mu / \mathrm{min}^{1 / 2}$ gesetzt wird.

Ferner erkennt man aus Abb. 2 b, daß für größere Längen $L$ als $\lambda_{\max }-b \sqrt{T}$ [Gl. (7)] die untere In- tegrationsgrenze nicht mehr $t=0$ ist, sondern durch den unteren Schnittpunkt $t=t_{1}$ der Geraden $L=$ const mit der Grenzkurve Gl. (6) gegeben ist. Die obere Integrationsgrenze ist für alle Längen $0 \leqq L \leqq L_{\max }$ der obere Schnittpunkt $t_{2}$ der Geraden $L=$ const mit der Grenzkurve. Bei $L=L_{\max }$ ist $t_{1}=t_{2}=\left(b^{2} / a^{4}\right) L_{\max }^{2}$, wie sich mit $\mathrm{d} L / \mathrm{d} t=0$ aus Gl. (3) ergibt.

Aus Gl. (8) folgt für $L \leqq \lambda_{\max }-b \sqrt{T}$

$$
\mathrm{d} N=\int_{0}^{t_{2}} k \mathrm{~d} L \frac{c}{V t} e^{-k(L+b V T-b \sqrt{t})} \mathrm{d} t+\frac{c}{V t_{2}} e^{-k\left(L+b V T-b V t_{2}\right)} \mathrm{d} t_{2}
$$

oder mit $\mathrm{d} t_{2}=\frac{2 L V t_{2}}{b L-a^{2} V t_{2}} \mathrm{~d} L$ aus Gl. (3):

$$
\frac{\mathrm{d} N}{\mathrm{~d} L}=\frac{2 c}{b} \frac{e^{-k L}}{e^{k b V T}}\left[\int_{0}^{t_{2}} \frac{k b}{2 V t} e^{k b V t} \mathrm{~d} t+\frac{e^{k b V t_{2}}}{1-\frac{a^{2} V t_{2}}{b L}} \mid\right] .
$$

Entsprechend erhält man für $L \geqq \lambda_{\max }-b V T$ :

$$
\frac{\mathrm{d} N}{\mathrm{~d} L}=\frac{2 c}{b} \frac{e^{-k L}}{e^{k b V T}}\left[\int_{t_{1}}^{t_{2}} \frac{k b}{2 V t} e^{k b V t} \mathrm{~d} t+\frac{e^{k b V t_{1}}}{\left|1-\frac{a^{2} V t_{1}}{b L}\right|}+\frac{e^{k b V t_{2}}}{\left|1-\frac{a^{2} V t_{2}}{b L}\right|}\right] .
$$

Nach Integration folgt aus den Gln. (10a) und (10 b)

und

$$
\begin{aligned}
& \frac{\mathrm{d} N}{\mathrm{~d} L}=\frac{2 c}{b} \frac{e^{-k L}}{e^{k b V T}}\left[e^{k b V t_{2}}\left(1+\frac{1}{a^{2} V t_{2}}-1\right)-1\right] \\
& \frac{\mathrm{d} N}{\mathrm{~d} L}=\frac{2 c}{b} \frac{e^{-k L}}{e^{k b V} V}\left[e^{k b V t_{2}}\left(1+\frac{1}{a^{2} V t_{2}}-1\right)-e^{k b V t_{1}}\left(1+\frac{1}{\frac{a^{2} V t_{1}}{b L}-1}\right)\right] .
\end{aligned}
$$

Da mit $\mathrm{d} L / \mathrm{d} t=0$ für $L=L_{\max }$ nach Gl. (3) $a^{2} \sqrt{t_{1}}=a^{2} \sqrt{t_{2}}=b L$ ist, muß für alle $t_{2}$ wegen $\mathrm{d} L / \mathrm{d} t<0$ stets $a^{2} \sqrt{t_{2}}-b L>0$ und für alle $t_{1}$ wegen $\mathrm{d} L / \mathrm{d} t>0$ stets $a^{2} V t_{\mathbf{1}}-b L<0$ sein (vgl. Abb. $2 \mathrm{~b}$ ). Deshalb können in der Schreibweise der Gln. (11 a) und ( $11 \mathrm{~b})$ die vorher benutzten Betragsstriche wegfallen.

An der Stelle $L=\lambda_{\max }-b \sqrt{T}$, d. h. für $t_{1}=0$, hat die Längenverteilungsfunktion eine Unstetigkeitsstelle: Beim Übergang von Gl. (11 a) zu (11 b) springt der Wert der eckigen Klammer um +1 . Dies liegt daran, daß die neu hinzukommende Nadelzahl auf der Grenzkurve in Abb. 2 b wegen des in der Rechnung benutzten Ansatzes für die Keimbildungsgeschwindigkeit $\mathrm{d} n_{0} / \mathrm{d} t=c / \sqrt{t}$ für $t=0$ unendlich wird. Andererseits ist aber an dieser Stelle der Grenzkurve $\mathrm{d} t / \mathrm{d} L=0$, wie sich aus dem Zusammenhang von Abb. 2 b mit 2 a ergibt, so daß nur ein endlicher Zuwachs der Nadelzahl resultiert. Bei endlicher Keimbildungsgeschwindigkeit für $t=0$ würde die Verteilungskurve stetig verlaufen. Der Fehler, den man durch Verwendung des eigentlich nur für $t \neq 0$ gültigen Ansatzes für die Keimbildungsgeschwindigkeit begeht, ist jedoch sehr klein, wie man sich durch Einsetzen der empirischen Werte $a, b$ und $k$ überzeugen kann.

Um die Längenverteilung in der Form $\mathrm{d} N / N \mathrm{~d} L$ zu erhalten, muß noch die Gesamtzahl $N$ der beobachtbaren Nadeln berechnet werden. Diese ist die Differenz aus der Zahl der während der Oxydationsdauer $T$ gebildeten und der Anzahl der von der Oxydschicht ganz zugedeckten Nadeln (schraffiertes Gebiet in Abb. 2 a). Mit Gl. (I, 8) und (I, 5) folgt

$$
N=\int_{0}^{T} \frac{c}{\sqrt{t}} \mathrm{~d} t-\int_{0}^{T} \frac{c}{\sqrt{t}}\left[\int_{0}^{b(v T-\sqrt{t})} k e^{-k \lambda} \mathrm{d} \lambda\right] \mathrm{d} t
$$


und nach Ausführung der Integration

$N=\frac{2 c}{k b} \frac{1}{e^{k b V T}}\left(e^{k b V T}-1\right)=\frac{2 c}{k b}\left(1-e^{-k b V T}\right)$.

Setzt $\operatorname{man} b=0,5 \mu / \min ^{1 / 2}$ (s. S. 62) und die aus der beobachteten Längenverteilung (Abb. I, 5) in Ver- bindung mit Gl. (I, 10) folgenden Werte für $k$ in $\mathrm{Gl}$. (13) ein und vergleicht mit $N=2 c \sqrt{T}$ nach Gl. (I, 8), so findet man, daß bei $3 \mathrm{~min} 75 \%$, bei 10 min $77 \%$ und bei 30 min $79 \%$ aller Nadeln von der Oxydschicht zugedeckt werden.

Division von Gl. (11 a) und (11 b) durch Gl. (13) ergibt:

$$
\frac{\mathrm{d} N}{N \mathrm{~d} L}=\frac{k \cdot e^{-k L}}{e^{k} b \sqrt{T}-1}\left[e^{k b \sqrt{t_{2}}}\left(1+\frac{1}{\frac{a^{2} \sqrt{t_{2}}}{b L}-1}\right)-1\right]
$$

und

$$
\frac{\mathrm{d} N}{N \mathrm{~d} L}=\frac{k \cdot e^{-k L}}{e^{k b V T}-1}\left[e^{k b \sqrt{t_{2}}}\left(1+\frac{1}{\frac{a^{2} \sqrt{t_{2}}}{b L}-1}\right)-e^{k b \sqrt{t_{1}}}\left(1+\frac{1}{\frac{a^{2} \sqrt{t_{1}}}{b L}-1}\right)\right] .
$$

Gl. (14 a) gilt für $L \leqq \lambda_{\max }-b \sqrt{T}$ und Gl. (14 b) für $L \geqq \lambda_{\max }-b \sqrt{T}$.

Die vorangegangenen Rechnungen gingen von der vereinfachenden Voraussetzung aus, daß alle Nadeln senkrecht zur Schichtoberfläche wachsen. Angenähert trifft dies auch zu: meistens sind die $\mathrm{Na}$ deln um Winkel zwischen $60^{\circ}$ und $90^{\circ}$ gegen die Schichtebene geneigt (sofern man bei der hügeligen Oberfläche der Oxydschicht überhaupt von einer Ebene sprechen kann). Bei diesen Winkeln ist aber die zusätzliche Verkürzung der Nadeln durch die Oxydschicht so geringfügig, daß sie die Längenverteilung nicht wesentlich beeinflussen kann. Flachere Neigungen als $45^{\circ}$ kommen nur vereinzelt vor.

Für die in der Verteilungskurve (Abb. I, 5) allein vorkommenden Längen $L<\frac{1}{2} L_{\max }$ ist $t_{2} \approx T$ (vgl. Abb. 2 b). Daher ist $a \sqrt{t_{2}} \approx a \sqrt{T}$ und wegen $a \approx 5 b$ nach Gl. (9) auch $\approx L_{\max }$. Da außerdem $L_{\max } \geqq 2 L$, ist in Gl. (14): $a^{2} \sqrt{t_{2}} / b L>10$, so daß sich die Längenverteilung mit sehr guter Annäherung durch

$$
\mathrm{d} N / N \mathrm{~d} L=k \cdot e^{-k L}
$$

in der gleichen Form wie nach Gl. (I, 10) darstellen läßt.

Während also die Gesamtnadelzahl $N$ ganz erheblich durch das Wachstum der Oxydschicht herabgesetzt wird, bleibt dieses ohne merklichen Einfluß auf die Längenverteilung der Nadeln.

In den folgenden Betrachtungen über die Nadellängenverteilung soll daher von einer Berücksichtigung des Schichtwachstums abgesehen werden.

\section{B. Die Abnahme der Inhibition mit der Oxydationszeit}

Wie in Abschnitt I, D. 1. schon dargelegt, würde Gl. (I, 10)

$$
\frac{\mathrm{d} N}{N \mathrm{~d} \lambda}=k e^{-k \lambda}
$$

den verschieden starken Abfall der Längenverteilungskurven (Abb. I, 5) dann wiedergeben, wenn der Inhibitionsfaktor $k$ nicht als konstant betrachtet wird, sondern mit fortschreitender Oxydationszeit abnimmt; das heißt $k$ ist eine Funktion von $t+\tau$ : $k=k(t+\tau)$.

Wird das Nadelwachstum durch Fremdstoffe blockiert, welche im Kupfer als Verunreinigung enthalten sind, so kann man die Zeitabhängigkeit von $k$ folgendermaßen erklären:

Ein Teil des Inhibitors bleibt in der $\mathrm{Cu}_{2} \mathrm{O}$ - bzw. CuO-Schicht stecken, etwa dadurch, daß er an Fehlstellen eingebaut wird. Außerdem ist auch eine Anlagerung an Kristallitgrenzen, Poren- oder Hohlraumwände innerhalb der Oxydschicht denkbar (vgl. z. B. Anm. ${ }^{3}$ ). Die im Schichtdickenelement $\mathrm{d} \delta$ absorbierte Inhibitormenge $\mathrm{d} M$ ist proportional der dort hindurchgehenden Menge $M$ :

$$
-\mathrm{d} M \sim M \mathrm{~d} \delta .
$$

Nach Integration folgt mit $\delta=b \sqrt{t+\tau}$ :

$$
M \sim e^{-B \sqrt{t+\tau}} .
$$

Da außerdem $M \sim k$ ist, kann man schreiben:

$$
k(t+\tau)=A \cdot e^{-B V \overline{t+\tau}} .
$$

Diese Beziehung ist auch gültig, wenn die Absorptionskoeffizienten im $\mathrm{CuO}$ und $\mathrm{Cu}_{2} \mathrm{O}$ verschieden sind, falls nur die Schichtdicken der beiden Oxyde während des Wachstums einander proportional blei-

3 A. Dravnieks u. H. J. McDonald, Trans. Elektrochem. J. 94, 139 [1948]. 
ben, was nach den Untersuchungen von Pä̈DAssi ${ }^{4}$ unterhalb von $750{ }^{\circ} \mathrm{C}$ zutrifft.

Als Ansatz für die Inhibition des Nadelwachstums gilt somit statt $\mathrm{Gl}$. (I, 2) :

$$
\mathrm{d}[\mathrm{d} n]=-A e^{-B v t+\tau} \mathrm{d} n \mathrm{~d} \lambda .
$$

$t+\tau$ ist die Zeit, zu welcher der Inhibitor an der Schichtoberfläche, d. h. am Fuß der Nadeln ankommt. Wie eine Abschätzung zeigt, ist die zur Dif- fusion an die Nadelspitze benötigte Zeit klein im Vergleich zur Wachstumszeit $\tau$ der Nadel und daher in Gl. (16) bzw. (17 a) vernachlässigt worden. Da aber auch die Zeit $\tau$ für die meisten Nadeln klein gegen die Zeit $t$ ist, vereinfacht sich Gl. (17 a) zu:

$$
\mathrm{d}[\mathrm{d} n]=-A e^{-B \sqrt{t}} \mathrm{~d} n \mathrm{~d} \lambda .
$$

Nach dem gleichen Rechnungsgang wie in Abschnitt I, D. 1. ergibt sich

$$
\mathrm{d} N=\frac{2 c \mathrm{~d} \lambda}{B \lambda} \int_{0}^{T-\tau} A \lambda \cdot e^{-B V \bar{T}} \cdot \frac{B}{2 \sqrt{t}} \cdot e^{-A \lambda \exp [-B V t]} \mathrm{d} t+\frac{c}{V T-\tau} e^{-A \lambda \exp [-B V T-\tau]} \mathrm{d} \tau .
$$

Da fast alle Nadeln im Bereich $\lambda \leqq \frac{1}{2} \lambda_{\max }=\frac{1}{2} a \sqrt{T}$ liegen und für diese $\tau \ll T$ ist, folgt mit $\mathrm{d} \tau=\left(2 \lambda / a^{2}\right) \mathrm{d} \lambda$ und $N=2 c \sqrt{T}$

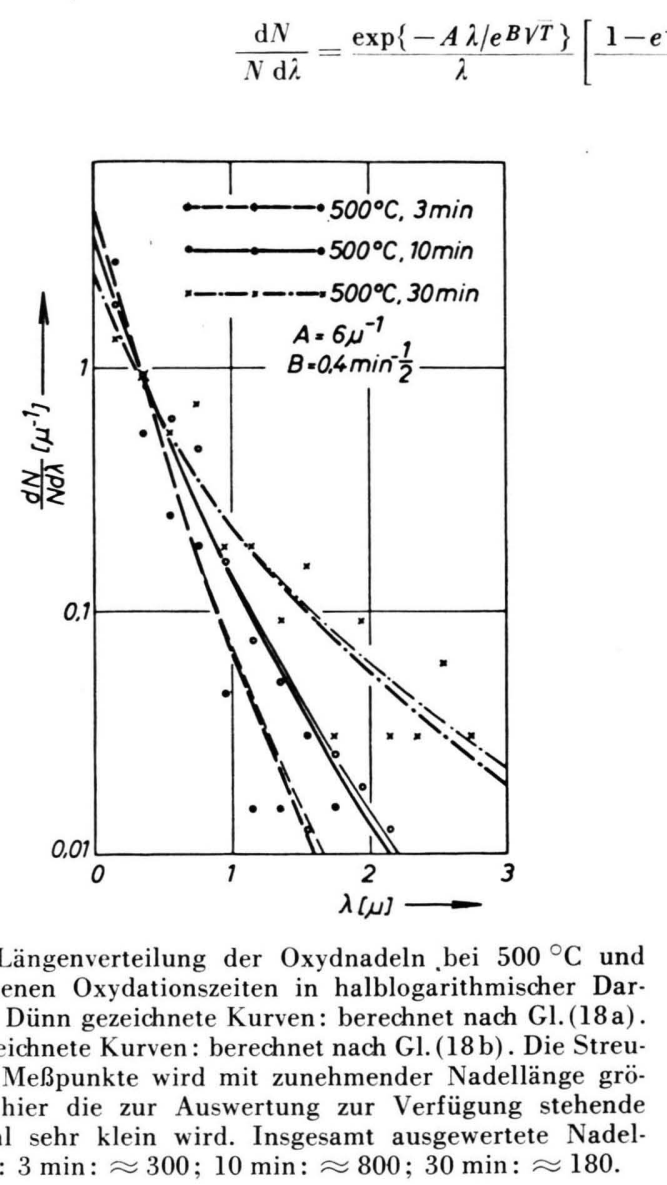

Abb. 3. Längenverteilung der Oxydnadeln bei $500{ }^{\circ} \mathrm{C}$ und verschiedenen Oxydationszeiten in halblogarithmischer Darstellung. Dünn gezeichnete Kurven: berechnet nach Gl.(18a). Dick gezeichnete Kurven : berechnet nach Gl. $(18 \mathrm{~b})$. Die Streuung der Meßpunkte wird mit zunehmender Nadellänge gröBer, da hier die zur Auswertung zur Verfügung stehende Nadelzahl sehr klein wird. Insgesamt ausgewertete Nadelzahlen: $3 \mathrm{~min}: \approx 300 ; 10 \mathrm{~min}: \approx 800 ; 30 \mathrm{~min}: \approx 180$.

Im betrachteten Längenbereich überwiegt der erste Summand in der Klammer und man erhält für die Längenverteilung mit zeitabhängiger Inhibition

$$
\frac{\mathrm{d} N}{N \mathrm{~d} \lambda}=\frac{1}{\lambda B V T}\left(e^{-A \lambda \exp [-B \gamma T]}-e^{-A \lambda}\right) .
$$

Der Abfall der Längenverteilungskurven mit der Nadellänge $\lambda$ hängt von $\operatorname{der} G$ röße der Konstanten $A$ und $B$ ab, während seine Abhängigkeit von der Versuchszeit $T$ allein durch $B$ bestimmt wird. Für $A=6 \mu^{-1}$ und $B=0,4 \min ^{-1 / 2}$ gibt Gl. (18 a) bzw. $(18 \mathrm{~b})$ die experimentell gefundene Längenverteilung gut wieder (vgl. Abb. 3).

\section{Einfluß von Dickenverteilung und Dicken- wachstum auf die Längenverteilung}

In den bisherigen Betrachtungen war der Einfachheit halber einheitliche Dicke für alle Nadeln angenommen worden. Wie die elektronenmikroskopischen Aufnahmen jedoch zeigen, liegt eine - wenn auch nicht sehr breite - Dickenverteilung vor. Sie kann davon herühren, daß bereits die Keime verschiedene Querschnitte besitzen und daß die Nadeln im Verlauf des Versuches dicker werden. Die Zeit $\tau$, wäh. rend welcher die Nadeln in die Länge wachsen, ist für den weitaus überwiegenden Teil der Nadeln klein gegen die bis zum Ende der Oxydation noch verbleibende Zeit, so daß das Dickenwachstum hauptsächlich erst im Anschluß an das Längenwachstum erfolgen wird. Dafür spricht auch die Beobachtung, daß die Nadeln bei $T=30 \mathrm{~min}$ im Mittel dicker sind als bei $T=3 \mathrm{~min}$. Ebenso beobachtete Pfefferkorn ${ }^{5}$ 
kein merkliches Dickenwachstum während des Längenwachstums der Nadeln.

Bei Vorliegen einer Dickenverteilung gilt für die Keimbildungsgeschwindigkeit statt Gl. (I, l a)

$$
\mathrm{d} n_{0}=\frac{c}{\sqrt{t}} \mathrm{~d} t f(q) \mathrm{d} q .
$$

$q$ ist der Nadelquerschnitt. Die Dickenverteilungskurve muß bei einem bestimmten Mindestquerschnitt $q_{0}$ beginnen (vgl. Anm. ${ }^{6,7}$ ) und kann qualitativ z. B. durch

$$
f(q) \sim \frac{q-q_{0}}{q_{0}} e^{-\alpha\left(q-q_{0}\right) / q_{0}}
$$

wiedergegeben werden (vgl. Abb. 4).

$\mathrm{Da}$ es von vornherein nicht auszuschließen ist, daß der Inhibitionsfaktor $k$ außer von $t$ auch von $q$ abhängig sein kann, gilt statt $\mathrm{Gl}$. (I, 2) :

$$
\mathrm{d}[\mathrm{d} n]=-k(q, t) \mathrm{d} n \mathrm{~d} \lambda .
$$

In Gl. (19) ist, wie im vorhergehenden Abschnitt, $\tau$ gegen $t$ vernachlässigt, also $t+\tau \approx t$ gesetzt worden; ferner wurde angenommen, daß, wenn $k$ überhaupt von $q$ abhängig ist, der für den Inhibitionsvorgang maßgebliche Nadelquerschnitt an der Spitze im Verlauf des Längenwachstums sich nicht ändert.

Wenn die Inhibition durch Fremdstoffe erfolgt

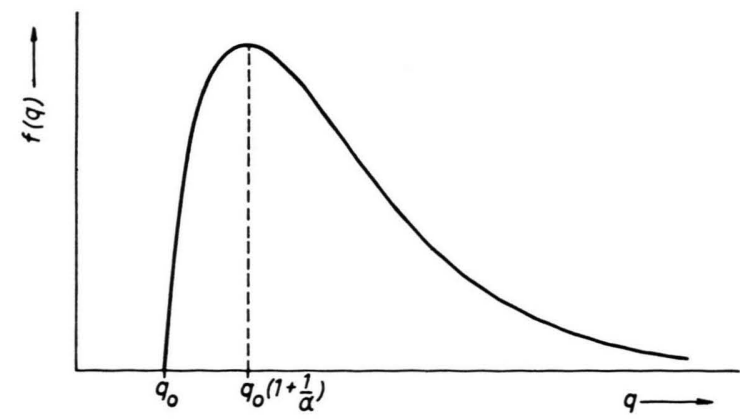

Abb. 4. Qualitative Darstellung der Dickenverteilung der Nadeln nach der Funktion $f(q) \sim\left[\left(q-q_{0}\right) / q_{0}\right] e^{-\alpha\left(q-q_{0}\right) / q_{0}}$.

und diese ebenso wie die Nadelsubstanz durch Oberflächendiffusion zur Nadelspitze gelangen, beeinflußt eine Verdickung des unteren Nadelteils, wie sie besonders bei langen Nadeln beobachtet wird, sogar während des Längenwachstums die Gültigkeit des Ansatzes (19) nicht. Denn Inhibitor und Nadelsubstanz diffundieren trotz veränderten Diffusionsquerschnittes stets im gleichen Verhältnis.

Die zum vorhergehenden Abschnitt analoge Durchrechnung ergibt mit dem durch Gl. (I, 3 b) gegebenen Wachstumsgesetz für Oberflächendiffusion $\tau=q \lambda^{2} / a_{s}{ }^{2} s$ die Längenverteilung

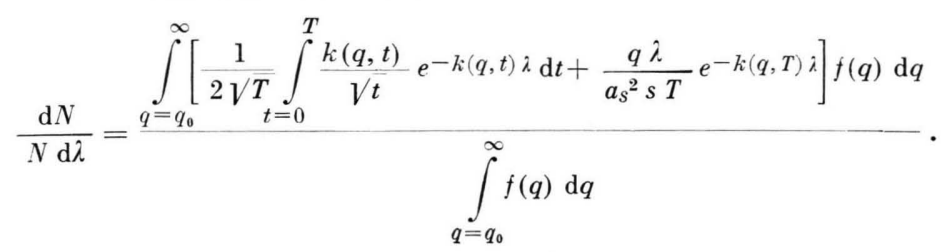

Aus dem gleichen Grund wie in Gl. (18 a) kann der zweite Summand im Zähler von Gl. (20) vernachlässigt werden. Für den Fall, daß $k$ von $q$ unabhängig ist, wie es bei Inhibition durch aus dem Metall stammende Fremdstoffe plausibel erscheint, geht Gl. (20) mit $k=k(t)=A e^{-B \sqrt{t}}$ in Gl. (18 b) über. Unter diesen Voraussetzungen hat also die Existenz einer Dickenverteilung keinerlei Einfluß auf die Längenverteilung.

\section{Schlußbemerkung}

Bei den vorhergehenden Überlegungen war stets vorausgesetzt, daß eine einmal erfolgte Inhibition des Nadelwachstums zu keiner späteren Zeit mehr aufgehoben wird. Inwieweit dies wirklich zutrifft, läßt sich elektronenmikroskopisch wegen der Objektverschmutzung im Mikroskop (vgl. z. B. TAKAGI ${ }^{8}$ ) nicht ohne weiteres entscheiden. Eine Möglichkeit bietet vielleicht die Methode von Heide ${ }^{9}$, mit der auch das Wachstumsgesetz der Einzelnadel besser untersucht werden könnte.

\footnotetext{
4 J. Pä̈dassi, Acta Metallurgica 6, 216 [1958].

5 G. Pfefferkorn, Z. wiss. Mikroskopie 62, 109 [1954].

6 F. C. Frank, Phil. Mag. (7) 44, 854 [1953].

7 P. B. Price, D. A. Vermilyea u. M. B. Webb, Acta Metallurgica 6, 524 [1958]

8 R. TAKagi, J. Phys. Soc., Japan 12, 1212 [1957].

${ }^{9}$ H. G. Heide, IV. Internationaler Kongre $\beta$ für Elektronenmikroskopie, Berlin, September 1958, Vortrag 11.09.
} 